\title{
DESEMPENho de Misturas B7 QuANTO A EMissões Veiculares E A ESTABILIDADE À OXIDAÇÃO
}

\author{
Tadeu Cavalcante Cordeiro de Melo ${ }^{1}$, Márcia Figueiredo Moreira ${ }^{1}$, Ivna Oliveira da Cruz ${ }^{1}$, \\ Mauro Iurk Rocha ${ }^{1}$, Maria Clara Kremer Faller ${ }^{1}$, Pedro Caffaro Vicentini ${ }^{1}$ e Aírton Giongo ${ }^{1}$, \\ ${ }^{1}$ Petrobras
}

E-mail: tcm@petrobras.com.br

\section{RESUMO}

Em 2013, houve o crescimento de discussões a nível governamental sobre a possibilidade do uso da mistura de 7\% v/v de biodiesel (B7) no óleo diesel nacional. Essa nova mistura óleo diesel-biodiesel substituiria a mistura B5 utilizada na época, no sentido de reduzir as importações de óleo diesel e de melhor aproveitar a capacidade industrial instalada dos produtores de biodiesel do país. Para se conhecer o desempenho da nova mistura B7, em comparação com a mistura B5, foram realizados diversos ensaios de emissões de poluentes em veículos e motores de diferentes tecnologias e fases do PROCONVE, em instalações laboratoriais de dinamômetro de chassi e de banco de provas, respectivamente. Foram utilizadas diferentes formulações de combustíveis, incluindo misturas de óleo diesel com teores de biodiesel de 5,7 e $10 \%$ v/v. Também foram conduzidos estudos referentes à estabilidade à oxidação da mistura B7, bem como a análise de outras propriedades físico químicas dessa nova mistura. Esse artigo apresenta os resultados de emissões veiculares das misturas B5, B7 e B10, bem como discussões sobre questões relacionadas à estabilidade à oxidação dessa nova mistura, que foi autorizada para uso no país a partir de 01 de novembro de 2014, lei $\mathrm{n}^{\circ}$ 13.033, de 24 setembro de 2014. O artigo também inclui comentários sobre o uso de misturas de biodiesel no Brasil, EUA e Europa, com ênfase no mercado americano e no mercado europeu.

\section{INTRODUÇÃO}

Em 2013, foram iniciadas discussões no Brasil para aumento do teor de biodiesel adicionado ao óleo diesel, que desde janeiro de 2010 estava em 5\% v/v. Como resultado do embasamento técnico de diversos trabalhos e estudos realizados pelo governo, indústria automotiva, ANP, centros de pesquisa e indústria de produção de biodiesel, foi adotada em maio de 2014 a Medida Provisória $n^{\circ}$ 647, que estabeleceu as datas de primeiro de julho e primeiro de novembro do mesmo ano para a adição obrigatória de biodiesel ao óleo diesel nos teores de $6 \%$ e $7 \%$ v/v, respectivamente [1]. A Lei 13033, de setembro de 2014, ratificou essa medida provisória e hoje todo o óleo diesel comercializado no Brasil conta com $7 \%$ v/v de biodiesel em sua composição [2].

Esse artigo apresenta os resultados laboratoriais de estabilidade à oxidação da mistura B7 e emissões em motores e veículos de diferentes tecnologias comparando as misturas B5, B7 e 
B10. De forma complementar também é apresentada uma revisão bibliográfica sobre legislações e uso de misturas de biodiesel ao óleo diesel no Brasil, Europa e EUA.

\section{REVISÃO BIBLIOGRÁFICA}

Nos Estados Unidos, o padrão "Renewable Fuel Standard" (RFS) estabelece que todo combustível utilizado no setor de transporte seja comercializado com um volume mínimo de combustível renovável [3].

De forma diferente do que ocorre no Brasil, os estados americanos podem definir diferentes mandatos e incentivos para o uso de misturas de biodiesel. A associação comercial "National Biodiesel Board (NBB)" coordena o programa de certificação BQ 9000 para promover a qualidade industrial do biodiesel certificando os produtores e distribuidores, quanto às práticas de manuseio e estocagem de toda a cadeia. Nos últimos anos, houve uma sensível melhora na qualidade do biodiesel comercializado nos EUA, principalmente devido ao programa de certificação BQ 9000 e ao aprimoramento nas especificações do biodiesel B100 [4].

Em 2013, cerca de $85 \%$ do biodiesel comercializado recebeu esta certificação, o que contribuiu para uma maior aceitação do uso do biodiesel no mercado e ajudou na garantia da qualidade dos requisitos das normas de especificação da ASTM (American Society for Testing and Materials) $[5,6,7,8]$.

Os limites máximos de biodiesel, aceitos por diferentes montadoras nos diversos segmentos do mercado dos EUA (veículos agrícolas, pesados e leves) variam dependendo do percentual de biodiesel adotado, B100, B20 ou B5 [9].

Na Europa, o uso de misturas de B0 a B7 está autorizado sem que haja necessidade de informação complementar ao consumidor desde 2009, quando a resolução EN 590 foi publicada [10]. Desde 2010, todos os veículos novos vendidos na União Europeia são compatíveis com B7 e desde junho de 2008, misturas maiores que B7 podem ser usadas em frotas cativas [11]. A SMMT (The Society of Motor Manufacturers and Traders), uma instituição de fabricantes de motores do Reino Unido, afirmou em seu nome e em nome de seus membros, que é improvável que ocorra qualquer problema devido à mudança da mistura de combustível de B5 para B7, incluindo veículos produzidos antes de 2010 [12]. Em abril de 2014, a ACEA ("European Automobile Manufacturer's Association") comunicou que todos os membros aceitariam a mistura B7 (especificada pelo padrão EN 590) em todos os veículos antigos ou novos [13].

Diversos trabalhos já foram publicados quanto a emissões, desempenho e durabilidade em veículos e motores de misturas B5 a B20, porém não foram encontrados muitos trabalhos sobre estabilidade à oxidação. A seguir é apresentado um resumo de alguns desses trabalhos.

O trabalho "Avaliação de misturas óleo diesel-biodiesel até B20 - Estabilidades à estocagem, à oxidação e térmica" apresentou resultados de estabilidade quanto à estocagem, à oxidação e térmica de misturas de combustíveis contendo 5, 10 e 20\% v/v de biodiesel em amostras de óleo diesel S10 e S500. Em percentuais de 10\% em volume foi constatado que as misturas apresentaram comportamento estável, entretanto para o uso da mistura B20 serão necessários estudos adicionais para adequação da especificação do biodiesel e do óleo diesel [14]. 
O trabalho "The Influence of Biodiesel Fuel Quality on Modern Diesel Vehicle Performance" mostra que, para o biodiesel dentro da especificação, não foram encontrados problemas de desempenho nos veículos para mistura B20 e B100. Para os veículos que operaram com biodiesel fora de especificação foram constatados problemas operacionais de depósitos internos nos bicos injetores, depósitos na câmara de combustão, aprisionamento dos anéis dos pistões e entupimento dos filtros de combustível [15].

O trabalho "Effects of Biodiesel Fuels Upon Criteria Emissions" apresentou uma avaliação bibliográfica de cerca de 1000 trabalhos sobre o uso de misturas de biodiesel em diferentes tecnologias de motores. De uma forma geral a adição de biodiesel contribuiu para uma redução de até $40 \%$ de material particulado e um acréscimo de $10 \%$ para o NOx com o uso do B100 [16].

No trabalho "Emission and performance evaluation of a PROCONVE P7 (EURO V) working with $5 \%$ up to $100 \%$ Biodiesel blends" foram encontradas uma perda máxima de $1 \%$ de potência com o uso de misturas até B30. Com relação ao consumo específico de combustível, foi verificado um aumento de $3 \%$ para misturas de B30. Com relação às emissões de $\mathrm{NOx}$ houve aumento de $30 \%$ para a mistura B30 [17].

Diversos estudos sobre durabilidade foram feitos com misturas B20. De forma geral, não foram encontrados problemas de desgaste acentuado do motor, porém houve a recomendação de diversos trabalhos para a redução do intervalo de troca do óleo lubrificante do motor $[18,19,20,21,22,23]$.

Com base nos estudos apresentados, de forma geral, para misturas até B20 não foram encontradas variações significativas de emissões de poluentes, bem como problemas com relação à durabilidade de motores. Dessa forma não são esperados problemas de durabilidade de motor com o uso da mistura B7.

Com relação à estabilidade à oxidação, a grande participação de cadeias insaturadas do biodiesel produzido a partir de soja e algodão, o avanço na tecnologia de motores do ciclo de diesel, o aumento do teor de biodiesel adicionado ao óleo diesel e as mudanças na especificação do óleo diesel automotivo contribuem para a ocorrência de problemas provenientes da degradação do combustível.

Estudos voltados para a abordagem da estabilidade de misturas BX começaram a ser divulgados e publicados somente nos últimos anos, com o assunto ainda cercado de incertezas e com muito a ser investigado. Até o presente momento, não há registro da existência de trabalhos que efetivamente indiquem os mecanismos predominantes e as interações, relativos ao óleo diesel e/ou ao biodiesel, no processo de degradação de formulações de suas misturas. Entretanto, avaliações contemplando métodos padronizados tradicionalmente utilizados para a verificação da estabilidade de óleos diesel e biodiesel, mesmo que ainda escassas, já se encontram disponíveis, contribuindo para o início do entendimento das estabilidades à estocagem e à oxidação das misturas BX.

Estudos realizados pelo laboratório de pesquisas americano National Renewable Research Laboratory - NREL abordaram a estabilidade à estocagem de misturas através dos métodos ASTM D4625, ASTM D2274 e pela determinação do período de indução - Rancimat [24,25]. 
Os resultados obtidos suportariam a ideia de que a estabilidade do biodiesel B100 consistiria do principal fator afetando a estabilidade de misturas B5 e B20, independentemente da aromaticidade, do nível de enxofre ou da estabilidade do óleo diesel utilizado na formulação.

Já um trabalho recente de Karavalakis et al [26], trouxe maiores esclarecimentos quanto a participação do óleo diesel na estabilidade das misturas BX, abalando até certo ponto a teoria suportada pelos estudos do NREL. Esse estudo verificou que a estabilidade do biodiesel B100, em geral, era refletida na estabilidade da sua mistura ao óleo diesel, mas que havia ainda a influência de uma série de outros fatores, incluindo a composição do biodiesel, a presença de aditivos antioxidantes e o seu grau de oxidação. Além disto, uma forte correlação entre a concentração do biodiesel e a estabilidade da mistura foi observada, com o aumento do teor de biodiesel na mistura resultando na redução de sua estabilidade.

Também foi constatado que a estabilidade das misturas BX ainda pode ser afetada por certas características do óleo diesel, com o maior hidrotratamento para obtenção de baixos teores de enxofre piorando a estabilidade da mistura BX final. Na ausência de enxofre no óleo diesel base para atuar como antioxidante natural, a presença de aditivos antioxidantes mostrou forte efeito sobre a estabilidade da mistura. Verificou-se igualmente que o óleo diesel que continha correntes de craqueamento catalítico em sua formulação se mostrou menos estável que o óleo diesel gerado a partir do hidrotratamento somente de correntes de destilação direta.

No entanto, nem todos os trabalhos presentes na literatura apontam para a piora da estabilidade pela incorporação do biodiesel ao óleo diesel. Estudo recente de Mushrush et al [27] verificou a existência de sinergia positiva relativa à estabilidade do produto na adição de 10 e $20 \%$ de um biodiesel considerado de qualidade superior (produto Soygold®) a óleos diesel avaliados pela metodologia ASTM D5304. A quantidade de insolúveis formados mediante o envelhecimento das amostras se mostrou menor para as misturas que para os óleos diesel base, particularmente para os óleos diesel classificados como instáveis.

Portanto, para conhecer melhor o comportamento da mistura B7, tanto quanto à sua estabilidade à estocagem, quanto ao seu desempenho em motores e veículos, realizou-se um extenso conjunto de ensaios cujos resultados são apresentados neste trabalho.

\section{RESULTADOS DE EMISSÕES DE MISTURAS DE BIODIESEL EM MOTORES}

\subsection{Combustíveis}

Foram utilizadas três diferentes misturas de biodiesel, com 5\%,7\% e 10\% v/v em óleo diesel S500 (500 ppm de enxofre) para os ensaios de emissões com motores de tecnologia CONAMA P4 e P5. Para o motor com tecnologia CONAMA P7 foram utilizadas misturas com os mesmos teores de biodiesel, mas usando como base óleo diesel S10 (10 ppm de enxofre).

As Tabelas I e II apresentam um resumo das principais propriedades das misturas de biodiesel preparadas com óleo diesel S500 e S10 e com biodiesel atendendo a Resolução ANP nº14 de 2012, respectivamente. 
Tabela I: Resumo das propriedades das misturas de biodiesel com S500

\begin{tabular}{cc|ccc}
\hline Características & Método & B5_S500 & B7_S500 & B10_S500 \\
\hline Enxofre, $\mathrm{mg} / \mathrm{kg}$ & ASTM D5453 & 398 & 391 & 383 \\
Teor de biodiesel, \% v/v & NBR 15568/2008 & 5,1 & 7,3 & 10 \\
Massa específica a $20^{\circ} \mathrm{C}, \mathrm{kg} / \mathrm{m}^{3}$ & ASTM D4052 & 850,3 & 851,0 & 851,9 \\
\hline
\end{tabular}

Tabela II: Resumo das propriedades das misturas de biodiesel com S10

\begin{tabular}{cc|ccc}
\hline Características & Método & B5_S10 & B7_S10 & B10_S10 \\
\hline Enxofre, $\mathrm{mg} / \mathrm{kg}$ & ASTM D5453 & 4,8 & 4,6 & 4,5 \\
Teor de biodiesel, \% v/v & NBR 15568/2008 & 5,0 & 7,0 & 10 \\
Massa específica a 20 ${ }^{\circ} \mathrm{C}, \mathrm{kg} / \mathrm{m}^{3}$ & ASTM D4052 & 832,0 & 832,9 & 834,3 \\
\hline
\end{tabular}

2.2. Motores

A tabela III apresenta resumos das especificações técnicas dos motores CONAMA P4, P5 e P7 usados neste trabalho.

Tabela III: Resumo das especificações do motor CONAMA P4

\begin{tabular}{c|ccc}
\hline Emissões & $\begin{array}{c}\text { EURO II } \\
\text { (CONAMA P4) }\end{array}$ & $\begin{array}{c}\text { EURO III } \\
\text { (CONAMA P5) }\end{array}$ & $\begin{array}{c}\text { EURO V } \\
\text { (CONAMA P7) }\end{array}$ \\
\hline Configuração & 4 cilindros, em linha & 4 cilindros, em linha & 4 cilindros em linha \\
Cilindrada & $<5$ litros & $<5$ litros & $<5$ litros \\
Sistema de combustão & Injeção Direta & Injeção Direta & Injeção Direta \\
Sistema de Injeção & Bomba rotativa & Common Rail & Common Rail \\
Aspiração & Turbo Intercooler & Turbo Compressor & Turbo Compressor \\
Potência & $100 \mathrm{~kW}$ & $130 \mathrm{~kW}$ & $140 \mathrm{~kW}$ \\
\hline
\end{tabular}

2.3. Resultados dos ensaios de emissões

Foram realizadas pelo menos três repetições de cada ensaio, de forma a permitir o tratamento estatístico dos dados. Os resultados foram comparados através do método de análise de variâncias ANOVA. Foram feitas comparações de médias e foi usado o teste Fisher LSD para 95\% de nível de confiança. 


\subsubsection{Emissões em motor CONAMA P4}

Os ensaios de emissões do motor com tecnologia CONAMA P4 (EURO II) foram feitos de acordo com o ciclo R49 da norma ABNT NBR 14489 [28]. As médias dos resultados de emissões dos poluentes gasosos e do material particulado (MP) com os três combustíveis testados estão na tabela IV.

Tabela IV: Resultados de emissões - ciclo R49 - motor CONAMA P4

\begin{tabular}{c|c|c|c|c}
\hline Combustivel & $\boldsymbol{C O}^{*}$ & $\boldsymbol{T H C}^{*}$ & NOx $^{*}$ & $\boldsymbol{M P}^{*}$ \\
\hline B5 & 100,0 & 100,0 & 100,0 & 100,0 \\
B7 & 103,4 & 96,6 & 100,6 & 101,7 \\
B10 & 101,2 & 90,3 & 102,8 & 98,8 \\
\hline
\end{tabular}

* resultados normalizados considerando a mistura B5 como referência

O tratamento estatístico dos dados é apresentado na tabela V.

Tabela V: Diferença estatística - ciclo R49 - motor CONAMA P4

\begin{tabular}{c|cc}
\hline & $B 7 \times$ B5 & $B 10 \times$ B5 \\
\hline CO & $=$ & $=$ \\
HC & $=$ & $=$ \\
NOx & $=$ & $2.8 \%$ \\
MP & $=$ & $=$ \\
\hline
\end{tabular}

Com base na tabela $\mathrm{V}$, pode-se verificar que não houve diferença significativa das emissões de poluentes gasosos e de material particulado para as misturas de B5 e B7. Com relação à mistura B10, a emissão de NOx mostrou uma elevação de $2,8 \%$ quando comparada à mistura B5.

\subsubsection{Emissões em motor CONAMA P5}

Os ensaios de emissões foram feitos de acordo com o ciclo ESC da norma ABNT NBR 15634 [29]. Os resultados médios de emissões do motor CONAMA P5 (EURO III) com os três combustíveis testados (B5, B7 e B10) estão na tabela VI.

Tabela VI: Resultado de emissões - ciclo ESC - motor CONAMA P5

\begin{tabular}{c|c|c|c|c}
\hline Combustivel & $\boldsymbol{C O}^{*}$ & $\boldsymbol{T H C}^{*}$ & NOx $^{*}$ & $\boldsymbol{M P}^{*}$ \\
\hline B5 & 100,0 & 100,0 & 100,0 & 100,0 \\
B7 & 96,5 & 91,2 & 101,1 & 100,0 \\
B10 & 95,9 & 92,6 & 98,9 & 93,1 \\
\hline
\end{tabular}

* resultados normalizados considerando a mistura B5 como referência

Na tabela VII é apresentado o resultado da análise estatística. 
Tabela VII: Diferenças estatísticas - ciclo ESC - motor CONAMA P5

\begin{tabular}{c|cc}
\hline & $\boldsymbol{B 7} \boldsymbol{x}$ B5 & $\boldsymbol{B 1 0} \boldsymbol{x} \boldsymbol{B 5}$ \\
\hline CO & $=$ & $=$ \\
THC & $-8,8 \%$ & $-7,4 \%$ \\
NOx & $=$ & $=$ \\
MP & $=$ & $-6,9 \%$ \\
\hline
\end{tabular}

Com base na tabela VII pode-se verificar que a adição de biodiesel no percentual de 7\% v/v (B7) não provocou acréscimo de emissões de poluentes em relação à mistura B5. Com o uso da mistura B10 foram verificadas reduções de até 6,9\% para o MP.

\subsubsection{Emissões em motor CONAMA P7}

Os ensaios de emissões em motor CONAMA P7 foram feitos de acordo com a norma ABNT NBR 15634 [29], incluindo os ciclos do tipo estacionário (ESC) e transiente (ETC).

As médias dos resultados dos ensaios de emissões com o uso das misturas B5, B7 e B10 estão apresentadas nas tabelas VIII e IX.

Tabela VIII: Resultados de emissões - ciclo ESC - motor CONAMA P7

\section{ESC}

\begin{tabular}{c|c|c|c|c}
\hline Combustivel & CO $^{*}$ & HHC $^{*}$ & NOx $^{*}$ & MP $^{*}$ \\
\hline B5 & 100,0 & 100,0 & 100,0 & 100,0 \\
B7 & 88,0 & 100,0 & 102,2 & 92,9 \\
B10 & 83,1 & 97,7 & 102,1 & 92,9 \\
\hline
\end{tabular}

* resultados normalizados considerando a mistura B5 como referência

Tabela IX: Resultados de emissões - ciclo ETC - motor CONAMA P7

ETC

\begin{tabular}{c|c|c|c|c}
\hline Combustivel & $\boldsymbol{C O}^{*}$ & NMHC $^{*}$ & NOx $^{*}$ & $\boldsymbol{M P}^{*}$ \\
\hline B5 & 100,0 & 100,0 & 100,0 & 100,0 \\
B7 & 85,6 & 86,2 & 104,1 & 104,7 \\
B10 & 88,1 & 96,6 & 102,9 & 100,9 \\
\hline
\end{tabular}

* resultados normalizados considerando a mistura B5 como referência

Nas tabelas X e XI são apresentadas em resumo as comparações estatísticas para os ciclos ESC e ETC, respectivamente. 
Tabela X - Diferenças estatísticas - ciclo ESC - CONAMA P7

\begin{tabular}{c|cc}
\hline & B7 $\boldsymbol{x}$ B5 & $\boldsymbol{B 1 0} \boldsymbol{x}$ B5 \\
\hline CO & $-11,9 \%$ & $-16,5 \%$ \\
THC & $=$ & $=$ \\
NOx & $=$ & $=$ \\
MP & $=$ & $=$ \\
\hline
\end{tabular}

Tabela XI: Diferenças estatísticas para o ciclo ETC - motor CONAMA P7

\begin{tabular}{c|cc}
\hline & $\boldsymbol{B 7} \times \boldsymbol{x}$ B5 & $\boldsymbol{B 1 0} \times \mathbf{B 5}$ \\
\hline CO & $-14,6 \%$ & $-12,1 \%$ \\
NMHC & $=$ & $=$ \\
NOx & $=$ & $=$ \\
MP & $=$ & $=$ \\
\hline
\end{tabular}

Com base nas tabelas X e XI pode ser verificado que a adição de biodiesel nos teores de 7 e $10 \%$ v/v não provocou mudanças significativas nas emissões dos poluentes, com exceção do $\mathrm{CO}$, que apresentou redução de até $16,5 \%$ com a mistura B10, cabendo ressaltar que esse poluente possui valores muito abaixo dos limites da legislação para motores diesel.

\section{RESULTADOS DE EMISSÕES DE MISTURAS DE BIODIESEL EM VEÍCULO}

3.1. Combustíveis

\subsubsection{Veículo PROCONVE L3}

Foi utilizado um diesel de 50 ppm de enxofre (S50), além de biodiesel de sebo e soja adquiridos de fornecedores do mercado para preparo de misturas de biodiesel nos teores de 5\% (B5) e 20\% (B20). Os resultados das principais análises feitas para o diesel S50 e suas misturas com o biodiesel são apresentados na Tabela XII.

Tabela XII: Resumo das propriedades das misturas de biodiesel com óleo diesel S50

\begin{tabular}{cc|cc}
\hline Características & Método & B5_S50 & B20_S50 \\
\hline Teor de biodiesel, \% v/v & NBR 15568/2008 & 5,0 & 20,0 \\
Massa específica a $20^{\circ} \mathrm{C}, \mathrm{kg} / \mathrm{m}^{3}$ & ASTM D4052 & 839,8 & 846,0 \\
\hline
\end{tabular}

\subsubsection{Veículo PROCONVE L6}

Para os ensaios de emissões no veículo com tecnologia da fase L6 do PROCONVE foram usadas as mesmas misturas com 5\%, 7\% e 10\% v/v (B5, B7 e B10) em óleo diesel S10 dos ensaios em motores. Suas principais propriedades podem ser vistas na tabela II. 
3.2. Veículos

A tabela XIII a seguir apresenta as especificações técnicas resumidas dos veículos usados neste trabalho.

Tabela XIII - Dados técnicos resumidos dos veículos

\begin{tabular}{|c|c|c|c|}
\hline Emissões & $\begin{array}{l}\text { PROCONVE L3 } \\
\text { VEÍCULO } 1\end{array}$ & $\begin{array}{l}\text { PROCONVE L3 } \\
\text { VEÍCULO } 2\end{array}$ & PROCONVE L6 \\
\hline Sistema de combustão & Injeção Direta & Injeção Direta & Injeção Direta \\
\hline Sistema de Injeção & $\begin{array}{c}\text { Bomba } \\
\text { Distribuidora }\end{array}$ & $\begin{array}{c}\text { Bomba } \\
\text { Distribuidora }\end{array}$ & Common Rail \\
\hline Controle de Injeção & Mecânico & Eletrônico & Eletrônico \\
\hline Aspiração & Turbo Intercooler & Turbo Intercooler & Turbo Intercooler \\
\hline
\end{tabular}

\subsection{Ensaios de Emissões e Autonomia}

Para as emissões em banco de chassis foi utilizada a norma ABNT NBR 6601 [30], que determina os protocolos para a medição das emissões de hidrocarbonetos totais (THC), monóxido de carbono (CO), dióxido de carbono ( $\mathrm{CO} 2)$, óxidos de nitrogênio (NOx), metano $(\mathrm{CH} 4)$ e material particulado (MP).

Para possibilitar o tratamento estatístico dos dados foram realizados pelo menos três ensaios de emissões de poluentes em dinamômetro de chassis para o veículo fase PROCONVE L6. Os resultados dos ensaios foram submetidos a um teste de homocedasticidade de variância de suas médias (o teste verifica se as variâncias são homogêneas, para que possa ser aplicado o teste de ANOVA). Depois as médias foram comparadas através do método de análise de variâncias ANOVA. Foram feitas comparações das médias e foi usado o teste Fisher LSD para 95\% de nível de confiança.

\subsubsection{Resultados de emissões em veículo PROCONVE L3}

Estudo anterior [31] comparou as emissões de misturas B20 e B5 em dois veículos da fase PROCONVE L3. Um resumo da análise estatística dos resultados é apresentado na tabela XIV.

Tabela XIV - Diferenças estatísticas de emissões - veículos PROCONVE L3

\begin{tabular}{c|cc}
\hline \multicolumn{3}{|c}{ B20 $\boldsymbol{x}$ B5 } \\
\hline & $\begin{array}{c}\text { PROCONVE L3 } \\
\text { VEÍCULO 1 }\end{array}$ & $\begin{array}{c}\text { PROCONVE L3 } \\
\text { VEÍCULO 2 }\end{array}$ \\
\hline CO & $=$ & $=$ \\
NOx & $-6 \%$ & $=$ \\
NMHC & $=$ & $=$ \\
MP & $=$ & $=$ \\
\hline
\end{tabular}


Da tabela XIV pode ser verificado que o uso da mistura B20 não provocou variação significativa das emissões para ambos os modelos de veículos. A exceção foi o poluente NOx, que apresentou uma redução de $6 \%$ no veículo 1 . Esse veículo, por possuir uma tecnologia antiga (bomba injetora mecânica), mostrou-se mais sensível ao uso da mistura B20. Com relação ao uso de misturas B7, pode-se esperar a mesma tendência dos resultados de emissões.

\subsubsection{Resultados de emissões em veículo PROCONVE L6}

Os resultados médios dos ensaios de emissões em $\mathrm{g} / \mathrm{km}$ do veículo da fase PROCONVE L6 são apresentados na tabela XV.

Tabela XV - Resultados de emissões - veículo PROCONVE L6

\begin{tabular}{c|c|c|c|c}
\hline Combustivel & CO $^{*}$ & NOx $^{*}$ & NMHC $^{*}$ & $\boldsymbol{M P}^{*}$ \\
\hline B5 & 100,0 & 100,0 & 100,0 & 100,0 \\
B7 & 112,9 & 100,0 & 87,5 & 103,4 \\
B10 & 105,9 & 100,0 & 62,5 & 89,7 \\
\hline
\end{tabular}

Para se comparar o desempenho dos diferentes combustíveis foram feitas tabelas de comparação estatística, a exemplo da apresentada anteriormente para os ensaios em motor. Na tabela XVI é apresentado um resumo das comparações estatísticas considerando como referência o combustível B5.

Tabela XVI - Diferenças estatísticas de emissões - veículo PROCONVE L6

\begin{tabular}{c|cc}
\hline & $B 7 \times B 5$ & $B 10 \times B 5$ \\
\hline CO & $=$ & $=$ \\
NOx & $=$ & $=$ \\
NMHC & $=$ & $-37,5 \%$ \\
MP & $=$ & $=$ \\
\hline
\end{tabular}

Com base na tabela XVI pode-se afirmar que não foram encontradas diferenças significativas com o uso das misturas B7 e B10 para os resultados de CO, NOx e MP em relação à mistura $\mathrm{B} 5$. Para as emissões de não metano (NMHC) foram encontradas reduções de até $37,5 \%$.

\section{AVALIAÇÃO DA ESTABILIDADE À ESTOCAGEM DO B7}

O trabalho consistiu na avaliação da estabilidade à oxidação, durante o envelhecimento a $43^{\circ} \mathrm{C}$ de misturas B7, formuladas com óleo diesel S10 (B7-S10), por um período de três meses, com objetivo de verificar a influência da qualidade do biodiesel e do óleo diesel utilizado nas formulações de B7 e o efeito de um pré-envelhecimento na estabilidade dessas misturas. 


\subsection{Combustíveis e análises físico-químicas}

Para formular as amostras B7-S10, foram selecionadas três amostras de biodiesel (1, 2 e 3) e duas de óleo diesel S10 (J e K). Como premissa adotada, as amostras de óleo diesel e biodiesel deveriam atender a especificação ANP vigente [32,33], com exceção da estabilidade à oxidação do biodiesel. Duas amostras de biodiesel (1 e 2) apresentaram estabilidade à oxidação inferior ao limite mínimo de 8 horas $(7,5 \mathrm{~h}$ e $6,7 \mathrm{~h}$ ) e somente uma amostra, identificada como 3, era estável, com período de indução igual a 8,4 horas.

No estudo, foram considerados dois grupos de amostras, onde um dos grupos visa avaliar o efeito do pré-envelhecimento das formulações no decaimento da estabilidade à oxidação durante estocagem a $43^{\circ} \mathrm{C}$.

a) B7-S10: amostras recém-formuladas e estocadas a $43^{\circ} \mathrm{C}$

b) B7-S10 envelhecidas: amostras estocadas a $43^{\circ} \mathrm{C}$ após serem envelhecidas a $80^{\circ} \mathrm{C}$ por 3 dias

A tabela XVII apresenta a nomenclatura das formulações avaliadas e os tipos das análises realizadas nesse trabalho.

Tabela XVII - Formulações e análises realizadas

\begin{tabular}{l|c}
\multicolumn{1}{c|}{ Amostra } & \multicolumn{1}{c}{ Análise Físico-Química } \\
\hline B7-S10 J1 & \\
B7-S10 J1 envelhecido & \\
\hline B7-S10 J2 & \multirow{2}{*}{ Estabilidade à Oxidação a 110 ${ }^{\circ}$ C (EN 15751) } \\
B7-S10 J2 envelhecido & Número de Hidroperóxido (ASTM D3703) \\
\hline B7-S10 J3 & \\
B7-S10 J3 envelhecido & \\
\hline B7-S10 K1 & \\
B7-S10 K1 envelhecido & \\
\hline B7-S10 K3 & \\
B7-S10 K3 envelhecido &
\end{tabular}

As formulações $\mathrm{J} 1, \mathrm{~J} 2$ e $\mathrm{J} 3$ foram preparadas com o óleo diesel $\mathrm{J}$, diferenciando-se quanto ao tipo de biodiesel (1, 2 e 3). Da mesma forma, as misturas K1 e K3, possuem o mesmo óleo diesel (K) na sua formulação e biodiesel diferente (1 e 3). Cabe ressaltar que a mistura K2 não foi formulada.

O acompanhamento da estabilidade das formulações estocadas a $43^{\circ} \mathrm{C}$, por um período de três meses, consistiu na realização mensal das análises de Estabilidade à Oxidação a $110^{\circ} \mathrm{C}$ e Número de Hidroperóxido. 
A determinação da estabilidade à oxidação das misturas $\mathrm{BX}$ é feita pelo método analítico EN 15751 [34], que consiste no envelhecimento da amostra a $110^{\circ} \mathrm{C}$ sob fluxo constante de ar $(10 \mathrm{l} / \mathrm{min})$. Os gases efluentes são coletados em água destilada cuja condutividade é monitorada continuamente. A etapa de propagação da reação de oxidação é facilmente observada pelo súbito aumento da condutividade da solução. $\mathrm{O}$ tempo decorrido até este súbito aumento é chamado de período de indução e é expresso em horas. Na legislação brasileira vigente [33], o limite mínimo para este parâmetro é de 8 horas para amostra de biodiesel. Para misturas de óleo diesel com $8 \%$ a $20 \%$ em volume de biodiesel [32,35], para uso experimental, o limite mínimo permitido é de 20 horas.

O método de determinação do Número de Hidroperóxido, ASTM D3703 [36], mede o teor de agentes oxidantes presentes no combustível, que podem ter sido formados durante o processo de degradação, sendo um indicativo de mudanças relativas que ocorrem no combustível durante o uso sob condições oxidantes.

\subsection{Resultados e Discussões}

Na tabela XVIII está disposta a caracterização inicial das formulações B7-S10.

Tabela XVIII - Caracterização inicial das formulações avaliadas

\begin{tabular}{l|c|c|c|c|c}
\hline \multicolumn{1}{c|}{ Característica } & B7-S10 J1 & B 7-S10 J2 & B7-S10 J3 & B 7-S10 K1 & B 7-S10 K3 \\
\hline Aspecto & LII & LII & LII & LII & LII \\
$\begin{array}{l}\text { Teor de Água, ppm } \\
\text { ASTM D6304 }\end{array}$ & 65,7 & 72,3 & 68,3 & 73,2 & 63,7 \\
$\begin{array}{l}\text { Estabilidade à Oxidação a 110 }{ }^{\circ} \mathrm{C}, \mathrm{h} \\
\text { EN 15751 }\end{array}$ & 21,4 & 15,4 & 20,8 & 24,0 & 34,9 \\
$\begin{array}{l}\text { Núme ro de Hidroperóxido, mg/kg } \\
\text { ASTM D3703 }\end{array}$ & 30,73 & 57,90 & 26,05 & 13,16 & 4,87 \\
$\begin{array}{l}\text { Insolúveis Totais, mg/100ml } \\
\text { ASTM D5304 }\end{array}$ & 0,4 & 1,1 & 0,0 & 0,0 & 0,0 \\
\hline
\end{tabular}

LII - límpido e isento de impurezas

As misturas apresentaram-se límpidas e isentas de impurezas e baixo teor de água. Em relação à estabilidade, a formulação J2 apresentou período de indução inferior a 20 horas e teor de insolúveis totais e hidroperóxido mais elevados quando comparado com as demais amostras. Por outro lado, a mistura K3 mostrou-se mais estável, apresentando período de indução elevado e baixa formação de insolúveis totais e hidroperóxidos.

Os resultados da avaliação das formulações durante o envelhecimento na estufa a $43^{\circ} \mathrm{C}$ por um período de 3 meses estão dispostos nas figuras 1 e 2 . 


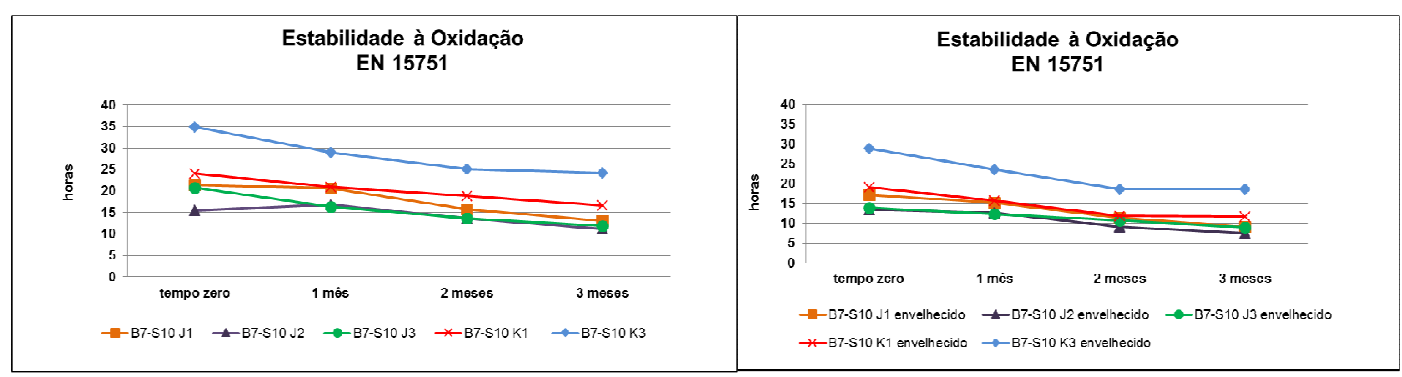

Figura 1 - Resultados de Estabilidade à Oxidação

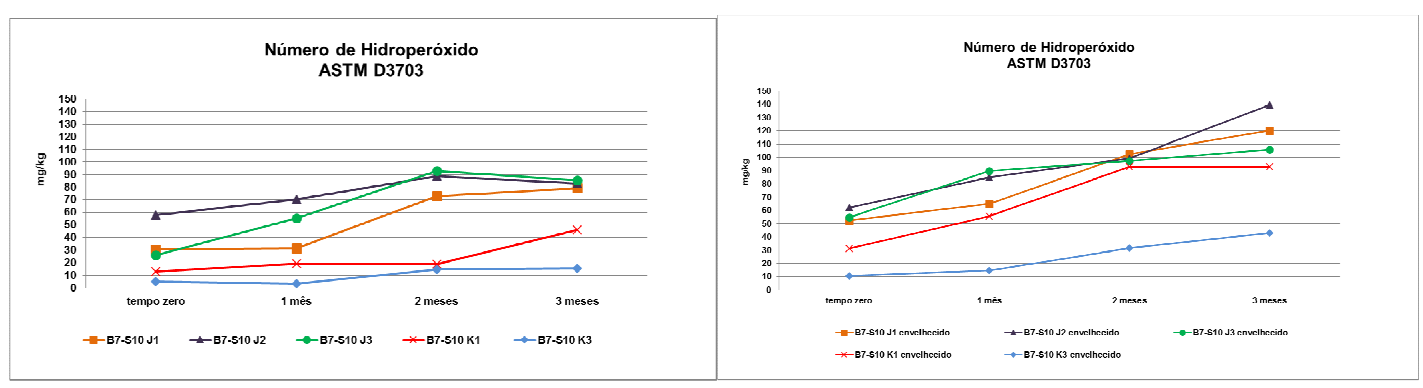

Figura 2 - Resultados de Número de Hidroperóxido

Todas as formulações de B7-S10 apresentaram redução da estabilidade e aumento do número de hidroperóxido ao longo do tempo.

As formulações com óleo diesel $\mathbf{J}$ apresentaram estabilidade à oxidação inferior às formuladas com óleo diesel $\mathrm{K}$ e formação de hidroperóxido mais acentuada durante $\mathrm{o}$ envelhecimento na estufa a $43^{\circ} \mathrm{C}$. Apesar das amostras de óleo diesel $\mathrm{J}$ e $\mathrm{K}$ atenderem à especificação ANP, em função da sua variabilidade química, foi observado um comportamento diferenciado em termos de decaimento da estabilidade à oxidação durante a estocagem, que pode ser atribuído à ocorrência de sinergias dos mecanismos oxidativos na mistura final.

Em relação à manutenção da estabilidade da amostra após três meses de estocagem, somente a mistura $\mathrm{K} 3$, formulada com biodiesel com período de indução superior ao limite mínimo de 8 horas, apresentou período de indução superior a 20 horas.

As demais amostras preparadas com biodiesel instáveis 1 e 2, com exceção da amostra $\mathrm{J} 2$, que, no tempo zero, já apresentou período de indução muito baixo, não mantiveram a estabilidade após três meses de estocagem, apesar de se mostraram estáveis no momento da formulação.

Em relação ao efeito do pré-envelhecimento das amostras antes da estocagem, apesar das mesmas apresentarem no tempo zero período de indução mais baixo, não foram observadas diferenças significativas no decaimento da estabilidade e na formação de hidroperóxidos ao longo do tempo quando comparado com as formulações recémformuladas. 


\section{CONCLUSÃO}

Com relação ao impacto sobre as emissões pela elevação do teor de biodiesel de $5 \%$ para $7 \%$ v/v na mistura com óleo diesel, foi verificado que:

- Em motores CONAMA P4, P5 e P7, não foram constatadas diferenças significativas nas emissões de NOx e de material particulado considerados os poluentes mais relevantes para motores diesel.

- Em veículos PROCONVE L3, estudo anterior comparando a mistura B20 com a mistura B5 em dois veículos diferentes mostrou que não houve variação significativa dos poluentes, com exceção de uma redução de $6 \%$ do NOx em veículo com tecnologia de bomba injetora mecânica, que se mostrou mais sensível ao tipo de combustível. Para uso de mistura B7, portanto, também não são esperadas variações significativas das emissões.

- Em veículo da fase PROCONVE L6 não foram encontradas diferenças significativas nas emissões de poluentes gasosos e de material particulado.

A comparação do uso da mistura B10 em relação à mistura B5 não revelou elevação das emissões tanto em motores quanto em veículos testados, com exceção do NOx que apresentou um acréscimo de 2,8\% nos resultados do motor CONAMA P7.

Os resultados de avaliação de estabilidade à oxidação quanto à estocagem para diferentes formulações de misturas B7 mostraram a manutenção de estabilidade à oxidação para um período de até três meses de estocagem.

Para o uso generalizado de misturas de biodiesel com teores acima de 7\% v/v são necessários estudos adicionais. Os estudos devem incluir, entre outros, uma verificação da adequação das propriedades físico-quimicas da nova mistura, dos métodos analíticos usados para análise, além de verificação da durabilidade e das emissões em motores.

\section{REFERÊNCIAS}

[1] Casa Civil, MEDIDA PROVISÓRIA no 647 de 28.05.2014. "Dispõe sobre a adição obrigatória de biodiesel ao óleo diesel comercializado ao consumidor final, e dá outras providências". Disponível em http://www.planalto.gov.br/ccivil_03/_Ato20112014/2014/Mpv/mpv647.htm .Acessado em 03.07.2014.

[2] Casa Civil, LEI no 13.033 de 24.09.2014. "Dispõe sobre a adição obrigatória de biodiesel ao óleo diesel comercializado com o consumidor final; altera as Leis $\mathrm{n}^{\mathrm{os}} 9.478$, de 6 de agosto de 1997, e 8.723, de 28 de outubro de 1993; revoga dispositivos da Lei no 11.097 , de 13 de janeiro de 2005; e dá outras providências". Disponível em http://www.planalto.gov.br/ccivil_03/_Ato2011-2014/2014/Lei/L13033.htm .Acessado em 28.05.2015.

[3] Renewable Fuels Standard (RFS) e Renewable Identification Number (RIN), EPA. Disponível em http://www.epa.gov/otaq/fuels/renewablefuels/ .Acessado em 08.08.2014. 
[4] NBB, BQ9000 - The National Biodiesel Accreditation Program. Disponível em http://www.bq-9000.org/.Acessado em 08.08.2014.

[5] ASTM D6751-12 - Standard Specification for Biodiesel Fuel Blend Stock (B100) for Middle Distillate Fuels, ASTM. Disponível em http://www.astm.org/Standards/D6751.htm Acessado em 08.08.2014.

[6] ASTM D975 - Standard Specification for Diesel Fuel Oils, ASTM. Disponível em http://www.astm.org/DATABASE.CART/HISTORICAL/D975-93.htm • Acessado em 08.08.2014.

[7] ASTM D7467 - Standard Specification for Diesel Fuel Oil, Biodiesel Blend (B6 to B20), ASTM. Disponível em http://www.astm.org/Standards/D7467.htm. Acessado em 08.08.2014.

[8] ASTM D396 - Standard Specification for Fuel Oils, ASTM. Disponível em http://www.astm.org/database.cart/historical/D396-96.htm. Acessado em 08.08.2014.

[9] Vehicle Technologies Office, DOE/GO-102014-4420.July 2014.Disponível em , www.cleancities.energy.gov. Acessado em 08.08.2014.

[10] EN 590:2009 - Automotive fuels - Diesel - Requirements and test methods - Directive 2009/30/EC. Disponível em www.dieselnet.com. Acessado em 03.12.2013.

[11] Association of European Automobile Manufacturers (ACEA). Disponível em http://www.acea.be . Acessado em 03/12/2013.

[12] Society of Motor Manufacturers and Traders (SMMT). Disponível em http://www.smmt.co.uk . Acessado em 03/12/2013.

[13] ACEA position concerning diesel that might contain more than $7 \%$ FAME, 22/04/14.Disponível em www.acea.be. Acessado em 08.08.2014.

[14] FALLER, M. C. K., IURK, M. R. et al.. Cold Flow Properties of Biodiesel Blends up to B20. SAE Brasil 2014-36-0495, 2014, SP.

[15] LOPES, S. M., CUSHING, T.. The Influence of Biodiesel Fuel Quality on Modern Diesel Vehicle Performance. SAE Technical Paper 2012-01-0858, 2012.

[16] ROBBINS, C., HOEKMAN E. C., et al. Effects of Biodiesel Fuels Upon Criteria Emissions, SAE Technical Paper 2011-01-1943, 2011.

[17] MACHADO, A. G., SHIRAIWA, N. M.. Emission and Performance Evaluation of a PROCONVE P7 (Euro V) Engine Working with 5\% up to $100 \%$ of Biodiesel Blends. SAE Technical Paper 2013-36-0208, 2013.

[18] MOREIRA, M.F., MAIA, D. M.. Impacto do Uso de Mistura com 20\% de Biodiesel em Diesel (B20) em Veículos. SIMEA 2014 PAP60, SP.

[19] FETRANSPOR - Biodiesel B20 - O Rio de Janeiro anda na frente. Rio de Janeiro, 2011. 
[20] BARBOSA, F. C.. Biodiesel Use in Transit Fleets - A Summary of Brazilian Experiences. SAE Technical Paper 2012-36-0149, 2012.

[21] BERMUDEZ, V., TORMOS, B., et al. Performance Evaluation and Components Behavior of Light Duty Diesel Engine after 300 Hours Test Fuelled with Pure Biodiesel: Effects on Reliability and Durability. SAE Technical Paper 2012-01-0865, 2012.

[22] LASTRES, L. F. M.. Efeito do Uso de B20 em Óleos Lubrificantes. SIMEA 2011, SP.

[23] WILlIAMS, A., McCORMICK, R. et al. Impact of Biodiesel Impurities on the Performance and Durability of DOC, DPF and SCR Technologies. SAE Technical Paper 2011-01-1136, 2011.

[24] MCCORMICK, R. L. e WESTBROOK, S. R. Storage Stability of Biodiesel and Biodiesel Blends. Energy Fuels, v. 24, p. 690-698, 2010.

[25] National Renewable Research Laboratory. Empirical Study of the Stability of Biodiesel and Biodiesel Blends. NREL/TP-540-41619 , 2007.

[26] KARAVALAKIS, G., STOURNAS, S. e KARONIS, D. Evaluation of The Stability of Diesel/Biodiesel Blends. Fuel, v. 89, p. 2483-2489, 2010.

[27] MUSHRUSH, G. W., HUGHES J. M. e WILLAUER H. D. Blends of Soybean Biodiesel with Petroleum Diesel: Advantages. American Chemical Society. Industrial \& Engineering Chemistry Research. v. 52. p. 1764-1768, 2013.

[28] ASSOCIAÇÃO BRASILEIRA DE NORMAS TÉCNICAS, Motor diesel - Análise e determinação dos gases e do material particulado emitidos por motores do ciclo diesel - Ciclo de 13 pontos - ABNT 14489, maio 2000.

[29] ASSOCIAÇÃO BRASILEIRA DE NORMAS TÉCNICAS, Veículos rodoviários automotores - Análise e determinação do gás de exaustão segundo os ciclos ETC, ESC e ELR - ABNT 15634, setembro 2009.

[30] ASSOCIAÇÃO BRASILEIRA DE NORMAS TÉCNICAS, Determinação de Hidrocarbonetos, Monóxido de Carbono, Óxidos de Nitrogênio e Dióxido de Carbono no Gás de Escapamento - ABNT NBR 6601, 2012.

[31] MELO, T. C. C., ROCHA, J. R. et al. Como Diferentes Teores de Biodiesel no Diesel Afetam as Emissões de Aldeídos em Motores e Veículos”, SIMEA, 2013,SP.

[32] Agência Nacional do Petróleo, Gás Natural e Biocombustíveis. RESOLUÇÃO ANP $\mathrm{N}^{\circ} 69$ de 23.12.2014.

[33] Agência Nacional do Petróleo, Gás Natural e Biocombustíveis. RESOLUÇÃO ANP No 45 de 25.8.2014. 
[34] EUROPEAN COMMITTEE FOR STANDARDIZATION EN 15751 Automotive fuels - Fatty acid methyl esters (FAME) and blends with diesel fuel - Determination of oxidation stability by accelerated oxidation method. CEN, 2009.

[35] Agência Nacional do Petróleo, Gás Natural e Biocombustíveis. RESOLUÇÃO ANP Nº 2 de 12.1.2011.

[36] AMERICAN SOCIETY FOR TESTING AND MATERIALS D 3703 - 07: Standard Test Method for Hidroperoxide Number of Aviation Turbine Fuels, Gasoline and Diesel Fuels: ASTM International, 2014.

Para esclarecimentos adicionais, contatar:

Tadeu Cavalcante Cordeiro de Melo, email: tcm@ petrobras.com.br PETROBRAS/CENPES. Tel: 2121626724 